\title{
Mobile tablet-based therapies following stroke: a systematic scoping review protocol of attempted interventions and the challenges encountered
}

\author{
Michael Pugliese ${ }^{1 *}$, Dylan Johnson ${ }^{1}$, Dar Dowlatshahi ${ }^{2}$ and Tim Ramsay ${ }^{3}$
}

\begin{abstract}
Background: Stroke is a growing global epidemic limiting the ability of millions to function independently due to post-stroke deficits and complications. Although specialized stroke rehabilitation improves the recovery of functional abilities, accessing rehabilitation services has become increasingly challenging as the number of stroke survivors continues to increase and rehabilitation resources remain scarce. Mobile tablet-based therapies (MTBTs) may be a resource-efficient platform for providing stroke rehabilitation services. The feasibility and challenges of offering MTBTs to stroke survivors should be well understood before expensive, large-scale clinical trials are undertaken to study treatment efficacy.
\end{abstract}

Method: A systematic scoping review will be conducted to describe attempted MTBTs following stroke and the challenges encountered by survivors and study staff. Studies of interest will evaluate MTBTs offered to adult stroke patients in response to post-stroke complications or deficits. Journal databases, gray literature sources, clinical trial registries, relevant organizational websites, and reference lists of eligible studies will be searched to identify suitable studies. Study characteristics, barriers to care, methodological challenges, patient-reported outcomes, and health outcomes will be extracted to describe MTBTs and understand the challenges encountered in context. Results will be presented using descriptive statistics, tables, figures, and narrative description to summarize the scope of the field.

Discussion: Trends in MTBT feasibility and common challenges will be discussed to summarize major findings and highlight research gaps. Solutions to common challenges experienced by intervention participants and study staff will be proposed. Implications for the conduct of randomized clinical trials of MTBT efficacy and the appropriateness of a systematic review and meta-analysis of completed trials will be discussed.

Systematic review registration: $\mathrm{uO}$ Research (http://hdl.handle.net/10393/35696).

Keywords: Stroke rehabilitation, mHealth, iPad, Tablet computer, CVA, Disability

\section{Background}

\section{Rationale}

\section{The growing burden of stroke}

Stroke is a global epidemic affecting millions worldwide. It is the second leading cause of death [1] and the third leading cause of disability globally [2]. The financial

\footnotetext{
* Correspondence: mpugl038@uottawa.ca

${ }^{1}$ School of Epidemiology, Public Health, and Preventive Medicine, University of Ottawa, Alta Vista Campus, Room 101, 600 Peter Morand Crescent, Ottawa, ON K1G 5Z3, Canada

Full list of author information is available at the end of the article
}

impact is also immense, incurring billions of dollars in healthcare costs annually [3]. This enormous burden has continued to increase with a reported $68 \%$ rise in the absolute number of first-time incidences of strokes and an $84 \%$ increase in stroke survivors worldwide in 2010 when compared to 1990 [4]. Disability and massive healthcare costs are caused by the wide range of poststroke impairments and complications experienced by stroke survivors. Specialized stroke rehabilitation effectively improves recovery following stroke [5] with the greatest improvements occurring when therapy 
begins early post-stroke and is performed intensely [6]. Best practice recommends timely transfer of stroke survivors to well-staffed, specialized inpatient rehabilitation units with sufficient resources to offer the variety and intensity of therapy needed to reduce chances of death and improve functionality.

\section{Accessing stroke rehabilitation services}

Accessing early and intensive stroke rehabilitation is challenging. Rehabilitation is not consistently initiated in the acute setting [7], and only $16 \%$ of stroke survivors in Canada are transferred to inpatient rehabilitation centers when estimates suggest $40 \%$ of survivors would benefit [8]. In the USA, only $24 \%$ of patients are transferred to inpatient services and only after waiting an average of 27 days post-stroke [9]. Accessing outpatient and community care is also difficult [10] with not all patients having immediate access to outpatient and community rehabilitation services [11]. The poor availability of stroke rehabilitation services is thought to be due to a lack of therapists with expertise in stroke [7, 9-11]. Additionally, therapists reported being assigned between 10.5 and 56 beds per therapist in Ontario rehabilitation centers [12] suggesting current therapists are already overburdened and unable to provide the intensive therapy needed for improving recovery.

\section{Improving the accessibility of stroke rehabilitation using mobile tablets}

Over the past decade, there has been growing interest in harnessing technology to support stroke rehabilitation and provide adjunctive therapy. There is growing research suggesting the positive effects of gaming on neurological outcomes following stroke with home video game consoles and virtual reality in particular have been shown to improve upper limb function and performance on activities of daily living [13]. Mobile video games have become available for mobile tablets in the form of software applications (apps), and there has been growing interest in mobile tablet-based therapies (MTBTs) following stroke. MTBTs use apps running on mobile tablet computers to provide interventions to patients. MTBTs are separate from and do not include therapies delivered via smartphone, mobile phone, or non-mobile touchscreen tablet technologies which are considered different therapeutic platforms. There are a variety of apps either explicitly designed to offer therapy (Constant Therapy® for aphasia and cognitive impairments) or involving activities analogous to scenarios often used in stroke rehabilitation (memory and attention games, etc.). Survivors could use MTBTs to either supplement therapist-led rehabilitation or begin engaging early rehabilitation until therapist-led services are successfully accessed.

\section{Why is it important to do a review now?}

Despite their wide availability, relatively low cost, and technological power, much remains unknown about MTBTs including treatment efficacy. However, before attempting small- or large-scale randomized controlled trials (RCTs) of treatment efficacy, the feasibility and challenges of offering MTBTs following stroke should be well understood in order to improve the chances of conducting successful studies. The study of MTBTs following stroke is still relatively new, and to the best of our knowledge, there is no systematic review summarizing attempted MTBTs. This systematic scoping review will be the first in the topic area and can provide answers to key questions regarding the feasibility and challenges of MTBTs following stroke while also describing the breadth of the field, identifying gaps in research, and informing the conduct of future RCTs and the appropriateness of meta-analysis of completed RCTs.

\section{Objective}

The objective of the study protocol is to review the evidence for mobile tablet-based therapies (MTBTs) following stroke.

\section{Research questions}

1. What are the characteristics of attempted MTBTs following stroke in terms of targeted deficits and method of administration?

2. What barriers or adverse events related to the administration of MTBTs following stroke have been encountered by researchers, clinicians, caregivers, or participants?

3. What methodological challenges have been faced by studies of MTBTs following stroke?

\section{Methodology}

This protocol was developed primarily with the assistance of a published guideline for scoping reviews [14] and with PRISMA-P (Additional file 1) when necessary and appropriate [15].

\section{Inclusion/exclusion criteria}

Inclusion criteria (must meet all):

1. Adult stroke survivors (18 years or older) of any type (ischemic/hemorrhagic) or stage (acute/ chronic) in any setting.

2. Stroke survivors interacting with a mobile tablet (not a smartphone, mobile phone, or non-mobile touchscreen tablet) in response to a post-stroke deficit or complication for therapeutic purposes.

Exclusion criteria (exclude if meet one or more): 
1. The mobile tablet is primarily used by someone other than the stroke survivor for purposes unrelated to tablet-based therapy support.

2. The mobile tablet is used primarily for purposes other than therapy.

\section{Criteria explanation and elaboration \\ Population}

Only studies involving adult stroke survivors will be included; children are a separate population outside of the scope of the proposed review. There are no restrictions with regard to stroke type or stage as the field is expected to be heterogeneous in this regard. Studies involving a mixture of stroke and non-stroke participants will be included only if they have separately reported data about stroke participants.

\section{Intervention}

We define MTBTs as patient-driven therapies in which participants interact via touch, speech, or movement with mobile tablet devices in response to a deficit or complication. The tablet device should be the primary method of therapy delivery; however, therapies involving peripheral devices (devices other than the core tablet unit itself including smartphone, robotics, sensors, etc.) will be included if the mobile tablet is clearly the primary platform for delivering therapy. MTBTs do not include smartphones, mobile phones, or non-mobile touchscreen tablets. This distinction has been because mobile tablets offer the unique benefit of having a large touchscreen interface that is likely easier for stroke survivors, who often suffer from motor and cognitive deficits, to manipulate while still remaining easily portable. This portability could allow survivors to bring their MTBT with them across their continuum of care from the acute hospital setting shortly after their stroke to their discharge destination. The use of tablets as assistive devices by clinicians for the administration of therapy, or by patients for the primary purpose of screening, assessment, or data collection does not constitute a MTBT. Tele-rehab programs using tablets solely as a method of videoconferencing with participants will not be included as the therapy is not truly mobile tabletbased; the tablet is simply acting as a means of providing traditional therapist-driven treatment.

\section{Context}

There are no restrictions related to context as we are interested in interventions performed in all settings and geographical locations, administered in all languages, and delivered by all types of therapists or non-therapists. However, only English-language publications will be considered due to expensive translation costs.

\section{Comparator(s)}

There are no restrictions related to comparators (standard treatment, workbooks, desktop or laptop computers, smartphones, etc.) as we are interested in describing all attempted MTBTs following stroke regardless of comparisons to other therapies.

\section{Outcomes}

There are no restrictions with regard to study outcomes as we are primarily interested in attempted interventions, therapy barriers, and research challenges. However, considering the study goals and research questions, we are interested in study outcomes including but not limited to barriers to care, adverse events, protocol deviations, Research Ethics Board issues, recruitment rate, adherence rate, retention rate, and patient evaluations of MTBTs.

\section{Study designs}

There are no restrictions with regard to study design: case studies/series, prospective and retrospective cohort studies, and randomized or non-randomized controlled trials of all designs will be included. There will be no restrictions with regard to study timing as it is expected that studies will substantially vary in length and timing. Study protocols and conference abstracts will only be included if they contain pilot or preliminary results from a study whose data are otherwise unavailable from a fullstudy manuscript. Included studies will be clearly marked as full-text articles, protocols, and abstracts, accordingly.

\section{Other restrictions}

As mentioned above, the included studies will be restricted to those written in English. Although the first modern tablet computers were introduced in the early 2000s, the surge in popularity of tablet computers with the release of the first Apple iPad( 2010 is well known. Additionally, the mobile tablet computers which will eventually be included in future randomized controlled trials will likely continue to improve upon the capabilities of their counterparts presented in this review. Therefore, in order to collect information that will be most relevant to information future RCTs, searches will be restricted to include studies between 2010 to present.

\section{Information sources \\ Preliminary search}

A preliminary search of the literature using key terms related to stroke and mobile devices in MEDLINE (OVID interface) yielded a number of studies meeting our inclusion criteria. These articles were used to 
identify key words and build a search strategy with the aid of a health information librarian. One study author (MP) piloted the search strategy in MEDLINE to ensure the strategy successfully re-identified the studies used to build the search. The search strategy successfully identified these papers, and no further modifications were made to the strategy except those necessary to adapt the strategy to different database search interfaces.

\section{Database searches}

The following six databases will be searched: MEDLINE (OVID interface), EMBASE (OVID interface), PsycINFO (OVID interface), CINAHL, Cochrane Database, and Web of Science.

\section{Additional information sources}

1. A snowball search of relevant articles and reviews identified by the database search.

2. Organizational websites: Aphasia.org, American Stroke Association webpage, Heart and Stroke Foundation webpage, and Stroke Engine.

3. Clinical trial databases will also be searched for completed and ongoing studies: ClinialTrials.gov, the WHO International Clinical Trials Registry Platform, EU clinical trials database, and ISRCTN.

\section{Gray literature search}

A gray literature search will also be performed in order to find unpublished material using Google Scholar, the ProQuest Dissertation and Theses Database (Global and UK \& Ireland), and the OpenGrey European gray literature database. After a preliminary search of Google Scholar and ProQuest Dissertation Global, it was decided searches would be limited to the first 200 results as a compromise between conducting a robust search and exhausting resources as search results beyond the first 200 results appeared to be irrelevant [16].

\section{Database search strategy}

The search strategy presented in Table 1 will be used to search databases with Ovid interfaces and adapted to search databases using other search interfaces. All index database searches were restricted to between the years 2010 and present and English language.

\section{Study records}

\section{Data management}

Database search results will be downloaded and imported to reference management software (Endnote ${ }^{\mathrm{Tx}}$ X8) in order to search for duplicates [17]. After duplicates have been removed using software and manual identification, the database results will be uploaded into Covidence $\odot$, an online systematic review manager, where all title/abstract and full-text screening will take place [18].

\section{Selection process}

Two authors (MP and DJ) will independently screen collected articles in a two-stage process with the assistance of an article screening form (Table 2): co-screeners will (1) screen study titles and abstracts returned by database searches for potentially eligible studies and (2) screen full-text manuscripts to confirm eligibility. The screening form will be piloted on batches of 30 title/abstract pairings and refined until an inter-rater agreement, as measured by the Kappa statistic, of 0.80 or above is achieved [19]. Screening conflicts will be resolved through discussion between screeners or resolved by a third party (DD) if necessary during both stages of screening. Reasons for full-text study exclusion will be tracked and listed. Screeners will not be blinded to study authors, affiliated institutions, or journal titles.

\section{Data collection process}

Two authors (MP and DJ) will independently extract key data items needed to describe the included studies and to answer the research questions stated above. A data extraction form (Additional file 2) will be used to guide data collection. Authors will compare and consolidate extracted information regularly to create a final data extraction form for each study. As the extraction process progresses, the data extraction form will be refined as necessary and any new pieces of information not collected in studies screened before changes occurred will be obtained by one of the study authors (MP or DJ). Study outcomes will be classified into one of three categories: barriers and adverse events, methodological challenges, and patient-reported outcomes. An assistive document (Table 3) will be used to help with the categorization of outcomes. Data extraction conflicts will

Table 1 PRISMA-P checklist

\begin{tabular}{ll}
\hline Search terms \\
\hline 1. & exp Stroke/ \\
2. & exp cerebrovascular disorders/ \\
3. & (stroke* or cerebrovascular* or cerebral vascular or CVA*).tw. \\
4. & ((cerebr* or brain) adj3 infarct*).tw. \\
5. & 1 or 2 or 3 or 4 \\
6. & (mobile device* or mobile computer* or handheld computer* \\
7. & (ipad* or galaxy tab* or surface pro*).tw. \\
8. & 6 or 7 \\
9. & 5 and 8 \\
\hline
\end{tabular}


Table 2 Article screening form

\begin{tabular}{|c|c|c|c|}
\hline Study element & Meets cr & & Reason for exclusion \\
\hline Population: Does the study enroll a population of human adults with stroke? & $\begin{array}{l}\text { Yes } \\
\text { Unclear }\end{array}$ & No & $\begin{array}{l}\text { 1. Not an adult population. } \\
\text { 2. Not a stroke population. }\end{array}$ \\
\hline $\begin{array}{l}\text { Intervention: Does the study involve stroke patients interacting with a } \\
\text { mobile tablet device in response to a post-stroke deficit or complication? }\end{array}$ & $\begin{array}{l}\text { Yes } \\
\text { Unclear }\end{array}$ & No & $\begin{array}{l}\text { 3. Not a tablet-based therapy. } \\
\text { 4. Survivors are not the primary tablet users. }\end{array}$ \\
\hline $\begin{array}{l}\text { Study Design: Does the manuscript report the results of a case study/series, } \\
\text { cohort study, randomized or non-randomized controlled trial? If a study } \\
\text { protocol or conference abstract, does it report the results of a study } \\
\text { whose data is not otherwise available in a study manuscript? }\end{array}$ & $\begin{array}{l}\text { Yes } \\
\text { Unclear }\end{array}$ & No & $\begin{array}{l}\text { 5. Manuscript is a protocol/conference abstract } \\
\text { with data available from a study manuscript. } \\
\text { 6. Manuscript is a protocol/conference abstract } \\
\text { with no reported data. }\end{array}$ \\
\hline
\end{tabular}

be resolved through discussion between screeners or resolved by a third party (DD) if necessary. No effort will be made to collect missing information from study authors due to time constraints.

\section{Data items}

A wide variety of data items are needed to adequately answer the proposed research questions in context. Although every effort has been made to anticipate the broad extent of variables which will be collected, refinements to collected information will be made as the data collection process progresses if deemed necessary. Data items of interest fall into six categories: general study information, participant characteristics, intervention details, comparator details, outcomes, and setting and context. A full list of variables and clarifications where necessary can be found in Additional file 2 .

\section{Outcomes and prioritization}

There is no need to prioritize outcomes to accomplish the objective of the review. All data items and outcomes of interest will be collected and discussed and holistically answer research questions and meet the study objective.

\section{Risk of bias in individual studies}

As per current scoping review guidelines, no formal risk of bias assessment will be performed for the included studies [14]. However, the potential impact of study

Table 3 Anticipated outcome categories of included studies

\begin{tabular}{ll}
\hline Outcome categorization \\
\hline $\begin{array}{l}\text { Outcome } \\
\text { category }\end{array}$ & $\begin{array}{l}\text { Outcome } \\
\text { sub-categories }\end{array}$ \\
\hline $\begin{array}{l}\text { Barriers and } \\
\text { adverse events }\end{array}$ & $\begin{array}{l}\text { Patient barriers, device barriers, environment } \\
\text { barriers, solutions to barriers (proposed/attempted, } \\
\text { and success if attempted), adverse events, other } \\
\text { barriers or possible adverse events }\end{array}$ \\
$\begin{array}{l}\text { Methodological } \\
\text { challenges }\end{array}$ & $\begin{array}{l}\text { Recruitment rate, adherence rate, retention rate } \\
\text { (loss to follow-up), reasons for dropout, reasons for } \\
\text { non-adherence, protocol deviations and/or revisions }\end{array}$ \\
$\begin{array}{l}\text { Patient-reported } \\
\text { outcomes }\end{array}$ & $\begin{array}{l}\text { Ratings of perceived usefulness of intervention, } \\
\text { other patient opinions. }\end{array}$ \\
\hline
\end{tabular}

design on individual study results will be discussed as the collected data items allow for an informed commentary.

\section{Data}

Development of outcome themes

The categories and sub-categories listed above (Table 3) will serve to guide the thematic development of qualitative outcome information relevant to the three research questions. Relevant qualitative information (patient/caregiver/physician interviews, reaction, options, etc.) will be entered into spreadsheet software by one reviewer (MP) and grouped into an appropriate outcome category and subcategory (Table 3) after final consolidated data extraction sheets for each study have been approved by both extractors (MP and DJ). The frequency of encountered themes will be noted in the presentation of the final results.

\section{Presentation of the final results}

Determining how to best present the results of a scoping review is an iterative process where the most logical approach becomes clearer as the data collection process comes closer to completion. Therefore, the following results outline is expected to be refined throughout the data extraction process. Search results will be summarized narratively and using the PRISMA flow diagram (Fig. 1) [20], and the characteristics of the final included studies will be summarized. The characteristics of the attempted intervention and administrative methods (therapy target, whether or not the therapy was performed with assistance, therapy setting, and whether the therapy was personalized) will be presented first to answer the first research question. This will be followed by lists of encountered barriers to care, adverse events, and other patientreported outcomes organized by categories listed above (Table 3) to answer the second research question. A list of methodological challenges reported by the included studies will follow answering the third and final research question. 


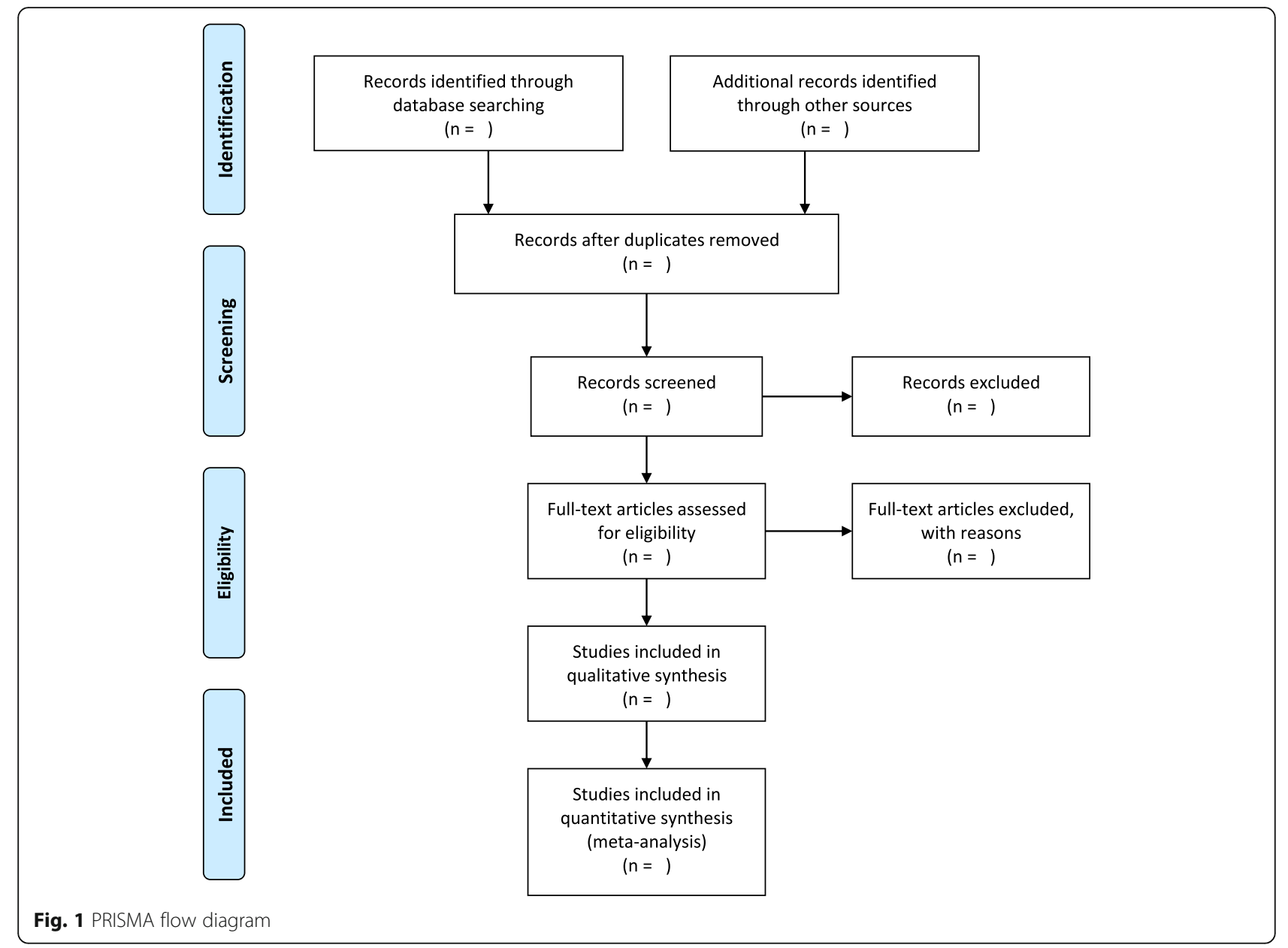

\section{Meta-bias(es) and confidence in the cumulative evidence}

There is no planned formal assessment of meta-biases or the confidence in the accumulated body of evidence. Instead, the impact of bias and strength of the evidence will be discussed based on the collected data points. More specifically, the strength of evidence supporting the feasibility of MTBTs following stroke will be covered in the discussion section of the final manuscript.

\section{Discussion}

Answers to review questions will be proposed based on the accumulated evidence. Solutions to common challenges faced by patients and researchers will be proposed, and if appropriate, recommendations will be made to evaluate these solutions. Limitations of the reviewed studies will be discussed, and recommendations for improving the design of observational studies of MTBTs will be made. Gaps in research in terms of under-studied patient populations, interventional areas, and settings will be addressed. Recommendations will be made for small- and large-scale randomized controlled trials of MTBTs, and comments will be made on the appropriateness of conducting a systematic review and meta-analysis of completed MTBT trials.

\section{Additional files}

Additional file 1: PRISMA-P checklist. (DOCX $28 \mathrm{~kb}$ )

Additional file 2: Data extraction form. (DOCX $13 \mathrm{~kb}$ )

\section{Abbreviations}

App(s): Application(s); MTBT: Mobile tablet-based therapy; RCT: Randomized controlled trial

\section{Acknowledgements}

The authors would like to thank Mish Boutlet of the University of Ottawa Health Sciences library for assisting with the refinement of the database search strategy.

This protocol is for an original systematic scoping review, not an update to a previous review.

\section{Amendments}

Amendment to protocol previously registered on January 11, 2017 (http:// hdl.handle.net/10393/35696). uO Research does not allow authors to host updated protocols. Protocol changes will be tracked and described in the final study manuscript along with justifications.

Funding

Mr. Pugliese is supported by an Ontario Graduate Scholarship and Canadian Institutes of Health Research Graduate Scholarship.

Dr. Dowlatshahi is supported by a Heart and Stroke Foundation New Investigator Award and a University of Ottawa Department of Medicine Clinician-Scientist Chair. 
No other sources of funding nor sponsors supported the current review. The funding sources had no involvement in developing the study protocol.

\section{Availability of data and materials}

All data generated or analyzed during this study will be reported in the final published manuscript and its supplementary information files.

\section{Authors' contributions}

MP developed study protocol and performed preliminary searches. DJ developed the study protocol. DD developed the study protocol. TR developed the study protocol. All authors read and approved the final manuscript.

\section{Ethics approval and consent to participate}

Not applicable.

\section{Consent for publication}

Not applicable.

\section{Competing interests}

The authors declare that they have no competing interests.

\section{Publisher's Note}

Springer Nature remains neutral with regard to jurisdictional claims in published maps and institutional affiliations.

\section{Author details}

${ }^{1}$ School of Epidemiology, Public Health, and Preventive Medicine, University of Ottawa, Alta Vista Campus, Room 101, 600 Peter Morand Crescent, Ottawa, ON K1G 5Z3, Canada. ${ }^{2}$ Department of Medicine (Neurology), University of Ottawa Brain and Mind Research Institute, and Ottawa Hospital Research Institute, C2182 Ottawa Hospital Civic Campus, 1053 Carling Avenue, Ottawa, ON K1Y 4E9, Canada. ${ }^{3}$ Ottawa Hospital Research Institute and Scientific Director at the Ottawa Methods Centre, University of Ottawa, Alta Vista Campus, 501 Smyth Rd, Ottawa, ON K1H 8L6, Canada.

Received: 17 February 2017 Accepted: 23 October 2017

Published online: 02 November 2017

\section{References}

1. Lozano R, Naghavi M, Foreman K, et al. Global and regional mortality from 235 causes of death for 20 age groups in 1990 and 2010: a systematic analysis for the Global Burden of Disease Study 2010. Lancet. 2010;380: 2095-128. https://doi.org/10.1016/S0140-6736(12)61728-0.

2. Murray CJL, Vos T, Lozano R, et al. Disability-adjusted life years (DALYS) for 291 diseases and injuries in 21 regions, 1990-2010: a systematic analysis for the Global Burden of Disease Study 2010. Lancet. 2012;380:1990-2010 https://doi.org/10.1016/S0140-6736(12)61689-4

3. Di Carlo A. Human and economic burden of stroke. Age Ageing. 2009:38:45. https://doi.org/10.1093/ageing/afn282.

4. Feigin VL, Forouzanfar MH, Krishnamurthi R, et al. Global and regional burden of stroke during 1990-2010: findings from the Global Burden of Disease Study 2010. Lancet. 2014;383:245-55. https://doi.org/10.1016/S01406736(13)61953-4

5. Teasell R, Foley N, Hussein N, Cotoi A. The efficacy of stroke rehabilitation. In: Evidence-based review of stroke rehabilitation. Heart and Stroke Foundation Canadian Partnership for Stroke Recovery. 2016. http://www.ebrsr.com/evidence-review/5-efficacy-stroke-rehabilitation. Accessed 3 Feb 2017

6. Teasell R, Hussein N. Background concepts in stroke rehabilitation. In: Evidence-based review of stroke rehabilitation. Heart and Stroke Foundation Canadian Partnership for Stroke Recovery. 2016. http://www. ebrrr.com/evidence-review/3-background-concepts-stroke-rehabilitation. Accessed 3 Feb 2017

7. Bernhardt J, Chan J, Nicola I, Collier JM. Little therapy, little physical activity: rehabilitation within the first 14 days of organized stroke unit care. J Rehabil Med. 2007;39:43-8. https://doi.org/10.2340/16501977-0013.

8. The Heart and Stroke Foundation 2014 stroke report. In: Canadian stroke best practice recommendations. The Heart and Stroke Foundation. 2014. http://www.strokebestpractices.ca/wp-content/uploads/2014/06/HSF_ SMReport2014E_Final.pdf. Posted June 2014. Accessed 3 Feb 2016.
9. Wang H, Camicia M, Terdiman J, Hung YY, Sandel ME. Time to inpatient rehabilitation admission and functional outcomes of stroke patients. Am J Phys Med Rehabil. 2011;3:296-304. https://doi.org/10. 1016/j.pmrj.2010.12.018.

10. Time is Function: A report from the Consensus Panel on the Stroke Rehabilitation System to the Ministry of Health and Long-Term Care. In: Consensus Panel on the Stroke Rehabilitation System. 2007. http://www. tostroke.com/wp-content/uploads/2011/03/Rehab-Consensus-Panel-FinalReport.pdf. Accessed 18 Nov 2016.

11. Teassell R, Foley N, Richardson M, Allen L, Cotoi A. Outpatient stroke rehabilitation. In: Evidence-based review of stroke rehabilitation. Heart and Stroke Foundation Canadian Partnership for Stroke Recovery. 2016. http:// www.ebrsr.com/evidence-review/7-outpatient-stroke-rehabilitation. Accessed 19 Aug 2017.

12. Meyer M, Foley N, Pereira S, Salter K, Teasell R. Organized stroke rehabilitation in Canada: redefining our objectives. Top Stroke Rehabil. 2012 19(2):149-57. https://doi.org/10.1310/tsr1902-149.

13. Laver KE, George S, Thomas S, Deutsch JE, Crotty M. Virtual reality for stroke rehabilitation. Cochrane Database Syst Rev 2015 Issue 1. Art. No.: CD008349. DOI: https://doi.org/10.1002/14651858.CD008349.pub3.

14. Peters MDJ, Godfrey CM, Khalil H, Mcinerney P, Parker D, Soares CB. Guidance for conducting systematic scoping reviews. Int J Evid Based Healthc. 2015;13:141-6. https://doi.org/10.1097/XEB.0000000000000050.

15. Moher D, Shamseer L, Clarke M, et al. Preferred reporting items for systematic review and meta-analysis protocols (PRISMA-P) 2015 statement. Syst Rev. 2015:4(1):1. https://doi.org/10.1186/2046-4053-4-1.

16. Stevinson C, Lalor DA. Searching multiple databases for systematic reviews: added value or diminishing returns? Complement Ther Med. 2004;12:22832. https://doi.org/10.1016/j.ctim.2004.09.003.

17. Endnote X8 Reference Management Software. Clarivate Analytics, Copyright 2017 Thomson Reuters. http://endnote.com/. Accessed 3 Feb 2017.

18. Covidence systematic review online management tool. Teamsquare, Level 2, 520 Bourke Street, Melbourne, Victoria, 3004. https://www.covidence.org/. Accessed 3 Feb 2017.

19. McHugh ML. Interrater reliability: the kappa statistic. Biochem Med. 2012; 22(3):276-82.

20. Moher D, Liberati A, Tetzlaff J Altman DG The PRISMA Group. Preferred reporting items for systematic reviews and meta-analyses: the PRISMA statement. PLoS Med. 2009;6(7):e1000097. https://doi.org/10.1371/journal. pmed1000097.

\section{Submit your next manuscript to BioMed Central and we will help you at every step:}

- We accept pre-submission inquiries

- Our selector tool helps you to find the most relevant journal

- We provide round the clock customer support

- Convenient online submission

- Thorough peer review

- Inclusion in PubMed and all major indexing services

- Maximum visibility for your research

Submit your manuscript at www.biomedcentral.com/submit
) Biomed Central 